\title{
Introduction
}

\section{A Long-Term Study}

A new technical method using autologous bone plugs with unilateral oblique posterior interbody fusion (UOPIF) was researched and developed into a successful procedure. It is a minimally invasive alternative to spine instrumentation.

Patients were treated individually across a period of 18 years, from 1990 to 2008 . 197 patients received autologous bone plugs at fusion for lumbar instability.

Spinal fixation with instrumentation is nowadays common practice and is a popular option for the treatment of segmental instability of the lumbar vertebral column.

Even though foreign body fixation has improved success rates in many disciplines, problems arise which include back pain caused by the instrumentation itself and allergic reactions and infection arising from the implantation of foreign bodies. Also, there is the issue of the high financial costs of the spine fixation instrumentation procedure.

However, there are now demonstrably significant advantages in utilizing the osseous consolidation artificial 'block spine' approach without foreign substances but with a living plug from the patient's own body. 